\section{Emerging and Re-emerging Diseases}

Stephen L. Roberts

Department of Health Policy, London School of Economics, London, UK

\author{
Keywords \\ Emerging diseases $\cdot$ Health security \\ Pandemic $\cdot$ Surveillance $\cdot$ Zoonoses
}

\section{Definition}

As defined by the World Health Organization (2014), emerging infectious diseases (EIDs) are serious global public health threats. An emerging infectious disease is one that either has appeared and affected a population for the first time or has existed previously but is rapidly spreading, either in terms of the number of people getting infected or to new geographical areas. Many EIDs are zoonotic in origin, which means that the disease has emerged from an animal and crossed the species barrier to infect humans. Nipah virus, Crimean-Congo hemorrhagic fever, and avian influenza A (H5N1) are examples of diseases that have recently emerged.

By contrast, re-emerging infectious diseases are diseases that once were major health problems globally or in a particular country, and then declined dramatically, but are again becoming health problems for a significant proportion of the population (malaria and tuberculosis are examples) (National Institutes of Health, 2007).

\section{Introduction}

The first two decades of the twenty-first century has witnessed a cascading of emerging infectious diseases. The first of these emergent epidemics was the appearance of a novel coronavirus in Southeast China in the late 2002. Initially misdiagnosed as a strand of atypical pneumonia (deLisle 2009), the severe acute respiratory syndrome or SARS would come to represent the first globalized public health emergency of the twentyfirst century, rapidly spreading out of Guangdong Province across a globalized transport infrastructure, eventually infecting over 8000 persons, in 26 countries, across 5 continents, resulting in 774 deaths (Tatem et al. 2006, p. 5).

Since 2002, a flurry of new infections and diseases have continually emerged, increasing in severity and scope. These novel infections, expedited by accelerated processes of globalization, shifting human migratory patterns, climate change and environmental degradation, and ever-increasing socioeconomic inequities, include mutating strains of avian and human influenzas, as in the H5N1 outbreak in 2005 and the 2009 H1N1 "swine influenza" pandemic; the devastating 2014 Ebola virus epidemic in West Africa, which killed thousands and cost billions of dollars to eventually contain; the 2015 outbreak of the Middle East 
respiratory syndrome (MERS-CoV) in South Korea; and the emergence and global spread of the Zika virus from 2015 onward. What is more, in February 2018, Chinese health authorities confirmed officially the first ever human case of H7N4, a highly pathogenic influenza subtype which had occurred in Jiangsu Province, transmitted originally from poultry stock (WHO 2018a), prompting fears of a new global pandemic.

Over the past two decades, the rise of an "epidemic of epidemics" (Bartlett 2014), represented by the emergence of numerous new infectious diseases, has elevated the politics of health, population security, and disease to the "high politics" (Fidler 2005, p. 180) of foreign, security, and defense policies of state governments and intergovernmental agencies alike, including the World Health Organization (WHO).

In the years following the SARS public health emergency, there has been an intensified shift and reconfiguration of the scope of international security practices which has facilitated the rise of "global health security." In an era of highly networked, albeit vulnerable globalized systems, the security implications of infectious diseases for state and international security and stability are manifold. The failure of an effectively and timely response to the 2014 West Africa Ebola epidemic resulted in the deaths of over 11,000 people across Guinea, Liberia, and Sierra Leone (CDC 2017), while in 2015, over 1.5 million people were infected by the Zika virus in Brazil alone.

In addition to the devastating impacts on the health and sustainability of human communities, emergent and recurrent disease outbreaks of the twenty-first century have produced destabilizing economic implications for states, regions, and global economic circulation. From 1995 to 2008, the estimated economic losses of these types of public health emergencies globally have exceeded US\$ 120 billion (Budke et al. 2006). Moreover, a recent WHO report, compiled during the West African Ebola epidemic, projected future economic losses stemming from a hypothetical moderate global influenza pandemic to be in the estimated range of 500 billion USD, or $0.6 \%$ of global income (Fan et al. 2017).
This chapter situates and provides a detailed overview of the current security challenges of emerging and re-emerging infectious diseases. It will do so by first presenting a profile of current emergent infectious diseases and considers the scope, potentiality, and likelihood of these infections to transpire into globalized pandemic outbreaks. In situating these infectious disease outbreaks within contemporary contexts of global health security and pandemic vigilance, the chapter affords a range of recommendations which seek to strengthen the effective identification, response, and mitigation of highly infectious global disease outbreaks.

\section{Emerging and Re-emerging Diseases}

The history of populations, and the growth of states have always been intimately intertwined with the emergence and movement of infectious disease, pathogens, and new viruses. Over the course of centuries, the emergence of novel infections have produced devastating implications for economies, states and societies; from the catastrophic Black Death, which killed $75 \%$ of the European population in the 1300 s, to the 1918 H1N1 influenza pandemic which killed 5\% of the world's population, to the emergence of the Ebola, HIV/AIDS and Zika viruses in the late 20th century. Throughout the early twentieth century, the monitoring and regulation of known infectious diseases including cholera, polio, plague, and yellow fever occurring at national and global levels were administered primarily by state governments, working in tandem with intergovernmental health organizations, first the League of Nations and later the World Health Organization (WHO) following its foundation in 1948.

Increasingly, however, global efforts to regulate the spread of infectious diseases across populations and geographies were problematized from the mid-twentieth century onward by the acceleration of emerging infectious diseases (EID). Unlike the catalogue of established and known diseases of past eras, emerging infectious diseases were distinct in profile in a number of critical attributes which included new or 
previously unobserved infections which resulted from changes or evolutions of existing organisms, known or established infections which had spread to new geographic regions or had infected previously unexposed populations, unrecognized infections appearing in areas undergoing ecological transformation, or old infections re-emerging as a result of antimicrobial resistance in known agents or breakdowns in public health measures (CDC 2014). As highlighted in a declassified estimate by the US National Intelligence Council titled The Global Infectious Disease Threat and Its Implications for the United States, since 1973 at least 30 previously unknown disease agents have emerged and have been identified, including many for which there exists no effective cure, including HIV, Ebola, and other hemorrhagic fevers including Lassa and Marburg fevers, hepatitis $\mathrm{C}$, the Nipah virus, the West Nile virus, and highly pathogenic strains of avian influenza (cited in Elbe 2009, pp. 2-3).

Concurrently, as new viruses and pathogens have successively emerged in recent decades, established infections and diseases for which therapies or treatments have traditionally existed have re-emerged in particularly virulent new formations including multidrug-resistant tuberculosis (MDR-TB) and the emergence of highly contagious strands of both bubonic and pneumonic plague in Madagascar in 2017, which to date has killed over 200 people.

Moreover, amid transformations in processes of globalization, persistent and irretractable intrastate conflicts and civil wars, the targeting of civilian populations during periods of hostilities, and the destruction of health infrastructures and the targeting of medical personnel have rapidly facilitated the re-emergence of highly infectious disease outbreaks including the reintroduction of poliomyelitis in Syria during the ongoing Syrian Civil War (2011-), and an epidemic of cholera in Yemen, severely exacerbated by the latest round of the ongoing Yemeni Civil War (2015-).

The emergence of a highly infectious pathogen of pandemic potential thus represents one of the most complex and pressing security challenges of the early twenty-first century. Emergent diseases and pathogens with high pandemic potential have been highlighted by the WHO (2010) by the high level of transmissibility across populations and age groups, the lack of pre-existing immunity to an emergent new infection, as well as a lack or absence of existing vaccinations, therapies, or medical countermeasures, requisite to inoculate populations from further infections during episodes of epidemic or pandemic outbreaks. Critically, emergent infections and viruses that have caused past pandemics across human populations typically have originated from animal influenza viruses and are classified as zoonotic infections (ibid).

In an era of heightened pandemic vigilance, the profile, nature, and scope of emerging infectious diseases are varied and multiform. Subsequently, a range of infectious diseases, with the cited potential to transpire into significant public health emergencies, are presented within the following classifications of emerging and re-emerging infectious diseases: bacterial infections and viral infections.

\section{Bacterial Infections}

The prevalence of bacterial-borne infections across populations has had incredible historical impact on the human species (Center for Health Security 2018). The Black Death, which represented recurrent waves of epidemics of plague (bubonic, pneumonic, and septicemic) across Europe, which killed millions of Europeans from the thirteenth century onward and which remains endemic in many parts of the globe presently, including recent re-emergent outbreaks in the Democratic Republic of the Congo, Madagascar, and Peru, is caused by the bacterium Yersinia pestis, with transmission of the bacterial infection occurring at the interface of human/animal interaction.

What is more are recurrent and globalized epidemics of cholera: throughout history, epidemics of cholera have been caused by an infection of the water-borne bacterium Vibrio cholerae. In January 1991, an epidemic of cholera re-emerged in Peru, sparking the first reintroduction of the bacterial infection in the Western Hemisphere in nearly a century (Sepúlveda et al. 2006, p. 5). This was followed 
by severe and widespread epidemics of cholera in Haiti following the 2010 earthquake and in Yemen from 2015.

Tuberculosis, a long-established infectious disease caused by the bacterium Mycobacterium tuberculosis, continues to represent a further critical public health issue in endemic and re-emergent/multidrug-resistant forms around the world. The WHO in its Global Tuberculosis Report (2017a) reported an estimated burden of 10.4 million new global TB infections, and 1.7 million deaths, of which 400,000 of these deaths were due to the increased prevalence of multidrug-resistant strands of tuberculosis (MDR-TB).

Presently, however, several critical factors limit the probability that widespread bacterial infections including plague, cholera, and tuberculosis could rapidly transpire into a global pandemic episode. Significantly, at present, effective treatments and therapies exist for these bacteriumbased infections. While these infections continue to reoccur in less developed regions of the world, the development of antibacterial therapies, including sulfonamides and penicillin, has dramatically reduced the global prevalence of these infections and limit their overall pandemic potential (Center for Health Security 2018, p. 12). Moreover, the relatively slow rate of replication and mutation of these bacteria further reduce the scale at which such an infection could be transmitted. In contrast, a human infected with the hepatitis $\mathrm{C}$ virus produces trillions of virions per day, whereas the doubling time of Yersinia pestis, the cause of forms of plague, is $1.25 \mathrm{~h}$ (ibid).

Although the resurgence of increasingly multidrug-resistant bacterial infections, including MDR-TB, signal a potential future health crisis, established, and in many cases treatable, infections including tuberculosis do not currently represent a global health security challenge in the form of a rapidly emergent pandemic outbreak. Correspondingly, while large-scale outbreaks of cholera and plague in Yemen and Madagascar represent grave public health emergencies, these outbreaks are symptomatic of inherently inequitable economic, historical, political, and societal realities which continue to underpin global health security frameworks.

\section{Viral Infections}

In contrast to bacterium-based infections, a majority of emergent pathogens and infections which constitute pandemic or epidemic diseases of heightened risk are viral-based infections which include Ebola and associated hemorrhagic fevers (including Crimean-Congo, Lassa, and Marburg hemorrhagic fevers), influenza (pandemic, seasonal, zoonotic), the Middle East respiratory syndrome-related coronavirus (MERS), monkeypox, the severe acute respiratory syndrome (SARS), and the Zika virus (WHO 2018b).

Unlike bacterium-based infections, the emergence and proliferation of new viruses and viral pathogens represent high-risk vectors for potential global pandemics. For example, the emergence of many novel and deadly viruses since the midtwentieth century has occurred within a context in which effective vaccines and medical or pharmaceutical countermeasures have been lacking or absent, as illustrated by the emergence of the HIV lentivirus, the SARS and MERS coronaviruses, and the Ebola virus.

Critically, many emergent viruses which have been observed over the past decades have extremely high rates of replication, mutation, and variance. A study initiated by Guan et al. (2002) observed the emergence of multiple new genotypes of the H5N1 influenza virus in poultry and geese in retail markets in Hong Kong, problematizing initiatives to develop a general vaccine to limit the spread of pandemic human or zoonotic influenza.

Recent seminal research texts further have underscored the high-level pandemic risks associated with emergent viral infections, specifically RNA viruses, which have ribonucleic acid as genetic material rather than DNA-based viruses. Various RNA viruses have emerged in particularly virulent forms over the past decades, transpiring into critical public health emergencies, and include Ebola and other associated hemorrhagic fevers, HIV, influenza (human and zoonotic), MERS-CoV, poliomyelitis, and SARS. 
In a recent report by the Johns Hopkins Center for Health Security titled The Characteristics of Pandemic Pathogens (2018), influenza, in both human and zoonotic forms, was listed as the highest pandemic potential RNA virus, with the avian influenza subtype H7N9 constituting the greatest single influenza pandemic risk on the basis of data derived from historical outbreak patterns and viral characteristics (Center for Health Security 2018). The report assessment further listed RNA infections including coronaviruses (MERS-CoV and SARS) to be the most probable pathogenic agents to result in a future global catastrophic biological event (GCBE) (ibid).

\section{Steps Forward: Addressing Emerging and Re-emerging Diseases}

The wake of the 2014 West Africa Ebola epidemic has reinvigorated and galvanized global efforts to monitor and regulate the emergence and re-emergence of pathogens with pandemic potential and to effectively address and contain infectious disease outbreaks to limit global contagion. While the past public health emergencies of the early twenty-first century (such as SARS, H1N1, and Ebola) represent diverse and varied health histories, key elements of pandemic preparedness and response - including increased spending and investment in health security, the bolstering of routine and timely disease surveillance systems, and continued investments and support for the development and deployment of vaccines to protect populations from infection and limit contagion - should continue to inform and guide both short- and longer-term initiatives to regulate the spread of emergent and re-emergent infectious diseases.

\section{Spending}

Continued, sustainable, and long-term investments in population health and health security systems constitute a vital element in the regulation of emergent and re-emergent disease threats. Over the past two decades, a range of new health security agencies and institutions have been founded with financing and spending mechanisms addressing a range of infectious diseases including the President's Emergency Plan for AIDS Relief (PEPFAR), the Global Fund to Fight AIDS, Tuberculosis and Malaria, and the Global Health Security Agenda (GHSA).

In terms of funding and financial support, development assistance for health and infectious disease priorities internationally have also quadrupled from US\$5.59 billion in 1990 to US\$21.79 billion in 2007 (Fidler 2011, pp. 1-2). And yet, critically, large-scale investments in global health projects and initiatives over the past decades failed to effectively stem the emergence and rapid transmission of the West Africa Ebola epidemic and further revealed deficiencies in almost every aspect of global defenses against emerging diseases and potential pandemics (National Academy of Medicine 2015). Thus, in the wake of the worst epidemic of the twenty-first century, there is a critical need for strategic spending in areas of health systems strengthening in continuing to address emerging and re-emerging infectious diseases.

Following the Ebola outbreak, the Centers for Disease Control and Prevention (CDC 2018) has estimated that annual investments of US $\$ 4.5$ billion into strengthening health capacity in both high- and low-income states could significantly reduce the occurrence of pandemic outbreaks. Suggested areas for investment within this proposal include surveillance systems for rapid detection and reporting; laboratory networks to accurately identify causes of illnesses; the training of medical workforces to track, identify, and contain outbreaks; and further developing of emergency management systems to coordinate effective outbreak responses (ibid).

Continued strategic spending in health systems must also be met with broader investments and an understanding of the conditions of economy, politics, and health which intertwine in the lives of populations in many areas of the globe. Correspondingly, new financial investments in securing against global disease threats have now been further aligned and merged with health and development priorities including the acceleration of investments in the survival of women and children and investments in development and well-being potential as recommended by the National 
Academies of Sciences, Engineering, and Medicine's Health and Medicine Division (2017).

Following the West Africa Ebola epidemic, the WHO has launched the Contingency Fund for Emergencies (CFE) in 2015, a collective resource fund to support responses to disease outbreaks and humanitarian crises with health consequences. From 2015 to 2018, WHO member states contributed US\$ 60 million in contingency funding for emergent public health emergencies. In 2018, the WHO released US\$ 1 million to support ongoing efforts to respond to and contain an expanding outbreak of the Ebola virus in Equateur Province, Democratic Republic of the Congo.

\section{Surveillance}

The routine, continuous, and systematic practices of public health and infectious disease surveillance form the bedrock of outbreak and epidemic response to emergent and re-emergent disease threats (WHO 2017b). Infectious disease surveillance systems enable the detection of early warning signals of probable public health emergencies or disease outbreaks and inform health authorities and practitioners of the scope or magnitude of the outbreak.

In an era of emergent and re-emergent diseases, practices of disease surveillance have transformed considerably in light of digital and technological advancements. The rise of new digital health surveillance systems from the 1990s onward, including ProMED-mail, GPHIN, and HealthMap, has accelerated the detection of emergent health emergencies by collecting and processing vast reservoirs of online digital data which, when filtered and analyzed, may indicate the onset of epidemic or pandemic illnesses. These digital disease sentinels have been rapidly integrated into the WHO's Global Outbreak and Alert Response Network (GOARN), a technical and scientific network which provides response and expertise to emerging global health events. Moreover, new digital surveillance technologies have been widely cited for the early identification of critical health episodes of the early twenty-first century including initial online detections of SARS and Ebola in West Africa.
In continuing to address looming emergent and re-emergent disease threats in a globalized world, practices of disease surveillance must be optimized with advancements in digital disease detection alongside continued investments and support for on-the-ground practices of clinical surveillance and data collection, most critically in regions with a high disease burden or prevalence of epidemic illness. This current gap between digital disease indicator systems and shortfalls in traditional disease surveillance practices was illustrated once more during the early and critical days of the SARS and Ebola epidemics in 2002/ 2003 and in 2014. In both cases, despite the presence of responsive digital disease surveillance platforms, a local deficit in broader virological awareness and an absence of robust and integrated surveillance practices meant that misdiagnosis and uncertainty abounded during the critical moments of each epidemic in which infection rapidly spread.

In future initiatives to address emergent and re-emergent diseases, the enhancement of surveillance practices which integrate digital technologies and continued traditional surveillance processes is vital. The wider global dissemination and sharing of viral profiles and surveillance best practices including for RNA-based respiratory infections and hemorrhagic fevers would bolster the advanced identification of emergent infections of these diseases in areas where such infections previously were absent but have emerged.

Moreover, despite the high-risk posed by RNA coronaviruses including SARS and MERs-CoV to result in a significant future public health emergency, as of 2018, there exists no global systemic laboratory surveillance of coronavirus infections in humans (Center for Health Security 2018). Thus, further efforts to construct responsive and timely global health surveillance apparatuses must focus upon and integrate this class of particularly pandemic-prone infections.

Further health security efforts to bolster and enhance the practice of disease surveillance through digital or clinical mediums must additionally adhere to rigorous ethical guidelines which ensure data protection, privacy, and personal integrity in responding to emergent disease outbreaks. In an era of new digital data sources and 
sophisticated data extraction technologies, vigilance and care must be employed in order to protect the identity, health information, and other elements of personal data from disclosure during public health emergencies. While it is imperative that states transparently share and disseminate epidemic intelligence in response to emergent and re-emergent disease risks, equal care must be taken by governments to ensure that identifiable data sources are protected or anonymized. In an era of multiple and diffuse digital data sources for the tracking of emerging diseases, the WHO Guidelines on ethical issues in public health surveillance (2017) represents a vital starting point in contemporary efforts to regulate health surveillance sources and practices amid concerns of heightened pandemic risk.

\section{Vaccines}

The production of effective vaccines to counter emergent and re-emergent pandemic risks, specifically strains of the human influenza virus, has been recognized as a global health imperative by the World Health Organization. Of the novel infectious diseases which have emerged since the late twentieth century, Ebola, HIV, MERS$\mathrm{CoV}$, SARS, the Zika virus, as well as human and zoonotic influenza viruses (H1N1, H7N9, and the recently emergent $\mathrm{H} 3 \mathrm{~N} 2$ ) all represent viral pathogens for which no current vaccine is available or effective across population groups.

The development and availability of vaccines are considered critical components of disease prevention for emerging and re-emerging infectious diseases (Maslow 2017), and yet the unique and complex nature of many emergent viral infections continue to problematize research progress to develop effective and durable vaccinations against some of the most severe viral pathogens. The emergence of these complex infections often has been accompanied by a lack of knowledge or research of the pathogenesis, epidemiology, and the epidemic potential of new infectious agents (ibid).

In steps to further address emergent and re-emergent diseases, the timely development and accessibility of vaccines to counter RNAbased respiratory viruses, as recommended by the Johns Hopkins Center for Health Security (2018, pp. 20-21), should be pursued with increased priority. Given the complexity of such respiratory infections, combined with high levels of genetic mutation, variance, and virulence, investments in the development of vaccines which protect against the spread of respiratoryborne infections could significantly reduce the probability of these disease outbreaks to transpire into global public health emergencies. As of 2018, the Coalition for Epidemic Preparedness (CEPI) has selected the MERS-CoV for further vaccine design incentivization (ibid), while the National Institute of Allergy and Infectious Diseases (NIAID) has unveiled its strategic plan for the development of a vaccine which can protect populations against multiple influenza strains (NIH 2018).

In addition to increased expenditure and vaccination research for pathogens of high pandemic potential, the further developing of vaccines to address emergent infectious diseases must operate with foresight and consistency, and not only based on spiked public interest and/or investment during public health crises - as witnessed during the most recent epidemic of Ebola in West Africa. At the same time, momentum toward the recognition of vaccines against viral agents as global public health goods and their enshrinement as such by legitimate health authorities including the WHO and its agencies should seek to offset the profitdriven agenda-setting and proclivities of pharmaceutical corporations, often in charge of vaccine development. Again, the severity of the Ebola outbreak had been exacerbated by a traditional lack of investment by large pharmaceutical corporations in vaccine development, due to the geographic isolation of the virus in Central Africa.

Critically, future investments in research and development to address emergent disease threats must occur within nuanced and global processes which recognize the interconnected realities of economy, politics, and society on patterns of health, infection, and disease, with particular emphasis on low- and middle-income countries. Calls for the rapid development of vaccines for Ebola and Zika viruses became globally prominent only following the potential for both 
infections to cross-national and regional jurisdictions - however, irrespective of initial vaccine availability, it was in fact the weak and vulnerable health systems and marked socioeconomic inequities across regions of Brazil and West Africa which were highly conducive to the initial spread of both viruses. Both the Zika and Ebola epidemics illustrated not only the importance of protecting people's lives through disease control and the timely provision of antivirals but also of the essentiality of a strong healthcare system embedded in a prosperous economy and a peaceful society (Heymann et al. 2015).

\section{Conclusion}

This chapter has developed a thematic overview of the present state of emergent and re-emergent diseases. The chapter has subsequently emphasized coordinated global and regional leadership to formulate timely and proportionate responses to emergent disease threats, strategic and targeted spending and investment, the continued development of effective and widely available vaccines to counter disease outbreaks, as well as the utilization of broad and nuanced understandings of the correlated realities of health and disease, economy, politics, and society as imperatives in continued global health initiatives to regulate and contain emergent and re-emergent diseases.

\section{Cross-References}

Ebola
$>$ Epidemics
$>$ Health Security
$>$ Pandemic Flu/H1N1

\section{References}

Bartlett, J. (2014). An epidemic of epidemics. Medscape Infectious Diseases, pp. 1-6.

Budke, C., Deplazes, P., \& Torgerson, P. (2006). Global socioeconomic impact of cystic echinococcosis. Emerging Infectious Diseases, 12(2), 296-303.
Centers for Disease Control and Prevention [CDC]. (2014). Emerging infectious diseases. Available at: https://wwwnc.cdc.gov/eid/page/background-goals

Centers for Disease Control and Prevention [CDC]. (2017). Outbreaks chronology: Ebola virus disease. Available at: http://www.cdc.gov/vhf/ebola/outbreaks/ history/chronology.html

Centers for Disease Control and Prevention [CDC]. (2018). Why it matters: The pandemic threat. https://www.cdc.gov/globalhealth/healthprotection/fiel dupdates/winter-2017/why-it-matters.html

de Lisle, J. (2009). Taiwan in the world health assembly: A victory, with limits. Available at: http:/www.brookings. edu/research/opinions/2009/05/taiwan-delisle.

Elbe, S. (2009). Virus alert: Security, governmentality, and the AIDS pandemic. New York: Columbia University Press.

Fan, V., Jamison, D., \& Summers, L. (2017). Pandemic risk: How large are the expected losses. Bulletin of the World Health Organization, 96(2), 129-134.

Fidler, D. (2005). Health as foreign policy: Between principle and power. The Whitehead Journal of Diplomacy and International Relations, 525, 179-194.

Fidler, D. (2011). Rise and fall of global health as a foreign policy issue. Global Health Governance, 4(2), 1-12.

Guan, Y., Peiris, J. S. M., Lipatov, A. S., Ellis, T. M., Dyrting, K. C., Krauss, S., Zhang, L. J., Webster, R. G. and Shortridge, K. F. (2002). Emergence of multiple genotypes of $\mathrm{H} 5 \mathrm{~N} 1$ avian influenza viruses in Hong Kong SAR. Proceedings of the National Academy of Sciences, 99(13), 8950-8955.

Heymann, D. L., Chen, L., Takemi, K., Fidler, D. P., Tappero, J. W., Thomas, M. J., Kenyon, T. A., Frieden, T. R., Yach, D., Nishtar, S., Kalache, A., Olliaro, P. L., Horby, P., Torreele, E., Gostin, L. O., Ndomondo-Sigonda, M., Carpenter, D., Rushton, S., Lillywhite, L., Devkota, B., Koser, K., Yates, R., Dhillon, R. S., \& Rannan-Eliya, R. P. (2015). Global health security: The wider lessons from the west African Ebola virus disease epidemic. The Lancet, 385(9980), 1884-1901.

Johns Hopkins Bloomberg School of Public Health, Centre for Health Security. (2018). The characteristics of pandemic pathogens. Available at: http:/www.centerforhealthsecurity.org/about-thecenter/pressroom/press_releases/2018-05-10_characte ristics-of-pandemic-pathogens.html

Maslow, J. (2017). Vaccine development for emerging virulent infectious diseases. Vaccine, 35(41), 5437-5443.

National Academies of Sciences, Engineering, Medicine. (2017). Global health and the future role of the United States. Available at: http://nationalacademies.org/hmd/ reports/2017/global-health-and-the-future-role-of-theunited-states.aspx

National Academy of Medicine. (2015). The neglected dimension of global security: A framework to counter infectious disease crises. Washington, DC: 
National Academy of Medicine. https://doi.org/10.17 $226 / 21891$

National Institutes of Health. (2007). Understanding Emerging and Re-emerging Infectious Disease, NIH Curriculum Supplement Series. Available at: https:// www.ncbi.nlm.nih.gov/books/NBK20370/\#A379

National Instutites of Health. (2018). NIAID Unveils Strategic Plan for Developing a Universal Influenza Vaccine. Available at: https://www.niaid.nih.gov/ newsevents/niaid-unveils-strategic-plan-developinguniversal-influenza-vaccine

Sepúlveda, J., Valdespino, J. L., \& García-García, L. (2006). Cholera in Mexico: The paradoxical benefits of the last pandemic. International Journal of Infectious Diseases, 10(1), 4-13.

Tatem, A., Rogers, D., \& Hay, S. (2006). Global transport networks and infectious disease spread. Global Mapping of Infectious Diseases: Methods, Examples and Emerging Applications, 62, 293-343.

World Health Organization. (2010). What is a pandemic? Available at: http://www.who.int/csr/disease/swineflu/ frequently_asked_questions/pandemic/en/

World Health Organization. (2014). A brief guide to emerging infectious diseases and zoonoses. Available at: https://apps.who.int/iris/handle/10665/204722

World Health Organization. (2017a). Global tuberculosis report. Available at: http://www.who.int/tb/publica tions/global_report/en/
World Health Organization. (2017b). Guidelines on ethical issues in public health surveillance. Available at: http:/www.who.int/ethics/publications/public-health-s urveillance/en/

World Health Organization. (2018a). Human infection with avian influenza virus. Available at: http:/www.who.int/ csr/don/22-february-2018-ah7n4-china/en/

World Health Organization. (2018b). Pandemic and epidemic diseases. Available at: http://www.who.int/emer gencies/diseases/en/

\section{Further Reading}

Erbelding, E., Post, D., Stemmy, E., Roberts, P., Augustine-Deckhut, A., Ferguson, S., Paules, C., Graham, B., \& Fauci, A. (2018). A universal influenza vaccine: The strategic plan for the National Institute of Allergy and Infectious Diseases. The Journal of Infectious Diseases. https://doi.org/10.1093/infdis/jiy103.

Johns Hopkins Bloomberg School of Public Health, Centre for Health Security. (2018). The characteristics of pandemic pathogens. Available at: http://www.cent erforhealthsecurity.org/about-the-center/pressroom/pre ss_releases/2018-05-10_characteristics-of-pandemic-p athogens.html

World Health Organization. (2017). WHO guidelines on ethical issues in public health surveillance. Available at: http://www.who.int/ethics/publications/publichealth-surveillance/en/ 